\title{
Kunststoffgleitlager mit intelligenter Verschleißüberwachung
}

\author{
Norman Katzer*, Hagen Bankwitz, Jörg Hübler \\ Professur Intelligente Maschinensysteme, Hochschule Mittweida, Deutschland \\ * Korrespondenz: katzer@hs-mittweida.de
}

Erhalten am 8. Oktober 2020; Angenommen am 12. Oktober 2020; Online zugänglich seit 7. Dezember 2020

(C) 2020 by N. Katzer u.a. Dies ist ein Open-Access-Artikel, der unter der Creative Commons Attribution License (CC-BY 4.O) verbreitet wird, die die uneingeschränkte Nutzung, Verbreitung und Vervielfältigung in jedem Medium erlaubt, vorausgesetzt, das Originalwerk wird ordnungsgemäß zitiert.

\begin{abstract}
Die Zustandsüberwachung (Condition Monitoring) und die vorausschauende Wartung (Predicted Maintenance) gelten als Schlüsselinnovationen der Industrie 4.0. Im Zuge dessen arbeiten Forscher der Professur Intelligente Maschinensysteme an der Hochschule Mittweida an einem Kunststoffgleitlager, welches eine integrierte Sensorik besitzt, die dem Anwender die Überwachung von Betriebsdaten ermöglichen soll. Die aus einem elektrisch leitfähigen Kunststoff hergestellte Sensorik trägt dazu bei, dass in Echtzeit sowohl eine Aussage zur Lagertemperatur als auch zum Verschleißgrad des Lagers getroffen werden kann. Damit lassen sich Wartungsintervalle besser planen und ein prophylaktischer Austausch von noch gebrauchsfähigen Lagern kann vermieden und damit Kosten gesenkt werden. Dafür sind umfangreiche numerische Untersuchungen zum mechanischen, thermischen und elektrischen Verhalten der Sensorik bzw. des Gleitlagers mit Interaktion der Welle durchgeführt worden. Dies beinhaltet auch eine Auswahl möglicher Werkstoffe. Außerdem ist die Auswerteelektronik (Messwertverstärkung, Datenverarbeitung und Datenübermittlung) entsprechend den Anforderungen der Sensorik entwickelt wurden. In einem weiteren Schritt wurde ein Funktionsmuster des Kunststoffgleitlagers mittels FDM-Drucks hergestellt.
\end{abstract}

KEYWORDS Zustandsüberwachung, Condition Monitoring, Predicted Maintenance, Strukturintegrierte Sensorik, Gleitlager, Reibung, Verschleiß, Tribologie, vorausschauende Instandhaltung, Industrie 4.0

\section{Einleitung}

Kunststoffgleitlager (siehe Abbildung1) besitzen ein riesiges branchenübergreifendes Anwendungsgebiet wie zum Beispiel in der Agrartechnik, der Automobilindustrie, der Baumaschinenbranche und der Luftfahrtindustrie. Selbst in Küchenmaschinen oder im Fitnessbereich sind Kunststoffgleitlager ein alltäglicher Begleiter. Ihre Popularität verdanken sie dabei der kostengünstigen Herstellung, der Beständigkeit gegenüber einer Vielzahl von Medien, dem schmierungsfreien Betrieb und der Toleranz gegenüber ungleichmäßiger Lasteinleitung. Aufgrund der Optimierung der tribologischen Eigenschaften der Kunststoffe, verdrängen sie dabei auch immer häufiger metallische Lager. Die Entwicklung hin zu höherer Temperaturbeständigkeit, höherer Festigkeit und besserem Reibungsverhalten führt dazu, dass Kunststoffe mittlerweile auch in hochbelasteten Reibkontakten 
Anwendung finden. Als hochbelastet wird dabei die Kombination aus Gleitgeschwindigkeit, Flächenpressung und Umgebungstemperatur bezeichnet. [1]

Problematisch dabei ist die Tatsache, dass das Reibungs- und Verschleißverhalten der Kunststoffe unter Einsatzbedingungen immer noch nur ungenau vorherzusagen ist. Dies begründet sich in der starken Abhängigkeit der mechanischen Eigenschaften von der Temperatur, welche sich wiederum auf den Reibungswert und den Verschleiß auswirken. Da die Reibung im Anwendungsfall zu einem Wärmeeintrag in der Kontaktstelle führt, verändern sich aufgrund der Temperaturerhöhung auch die mechanischen Eigenschaften der Kontaktbereiche. Dies führt zu einem dynamischen Reibungs - und Verschleißverhalten, welches durch die schlechte Wärmeleitfähigkeit der Kunststoffe noch verstärkt wird. [2]

Infolgedessen lassen sich die Ergebnisse aus Laborversuchen nur selten auf den realen Anwendungsfall übertragen. Eine Übertragbarkeit ist nur dann gegeben, wenn die Beanspruchung und die Geometrie im Wesentlichen mit dem realen Anwendungsfall übereinstimmen. Eine exakte Lagerdimensionierung ist somit nicht möglich. Daraus resultiert, dass die Lebensdauer des Lagers nicht bestimmt werden kann und Austauschintervalle damit nicht auf einen konkreten Zeitpunkt festgesetzt werden können. Solche Intervalle müssen bei hochproduktiven Maschinen und Anlagen jedoch planbar sein, um Stillstandzeiten und damit verbundene hohe Kosten zu minimieren.

Im Zuge dessen soll ein sensorintegriertes Kunststoffgleitlager entwickelt werden, welches eine zustandsorientierte Instandhaltung ermöglicht. Im Gegensatz zu den bisher üblichen reaktiven (Austausch nach Defekt) und präventiven (Austausch nach festgelegtem Intervall) Instandhaltungsmethoden, können damit Folgekosten durch Lagerschäden vermieden und vorhandene Restlaufzeiten optimal ausgenutzt werden. Die zusätzlich gewonnene Betriebszeit führt $\mathrm{zu}$ einer gesteigerten technischen Verfügbarkeit des gesamten technischen Systems und damit letztlich auch zu einer Produktivitätssteigerung. [3]

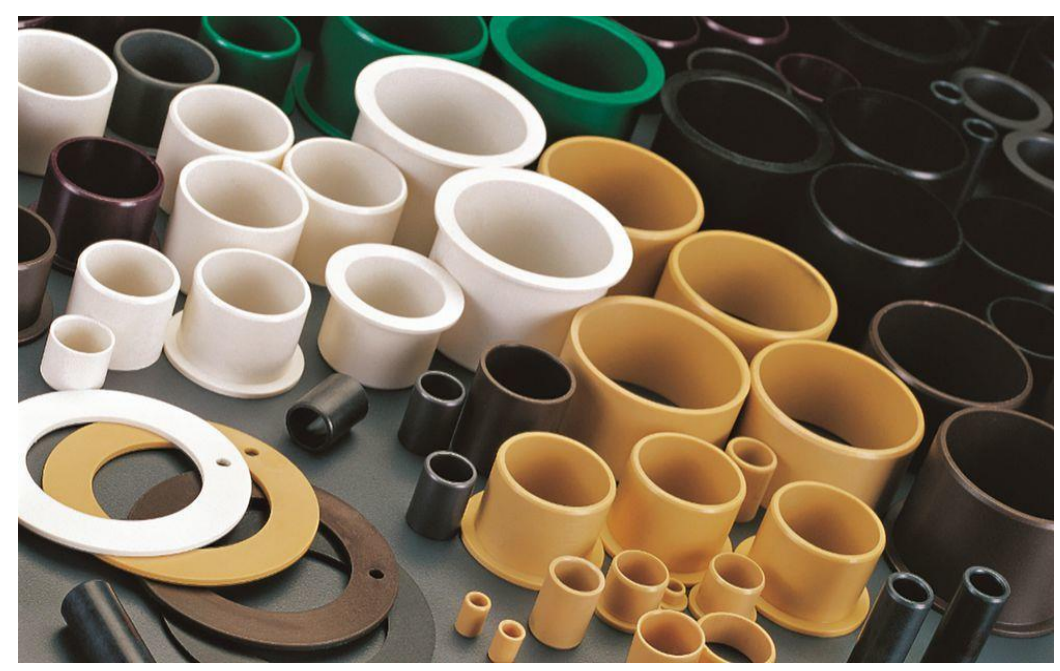

Abbildung 1: Kunststoffgleitlager [4] 


\section{Funktionsprinzip}

Das Kunststoffgleitlager besteht in seinem Grundaufbau (Abbildung 2) aus zwei unterschiedlichen Materialien. Eines der beiden Materialien (in der Abbildung rot dargestellt) besitzt elektrisch leitfähige Eigenschaften und wird daher als Sensorelement benutzt. Dieses Sensorelement wird in einem Spritzgussprozess in das zweite elektrisch nicht leitfähige Material eingebettet. Das zweite Material (grün) dient als Verschleißschicht mit guten tribologischen Eigenschaften.

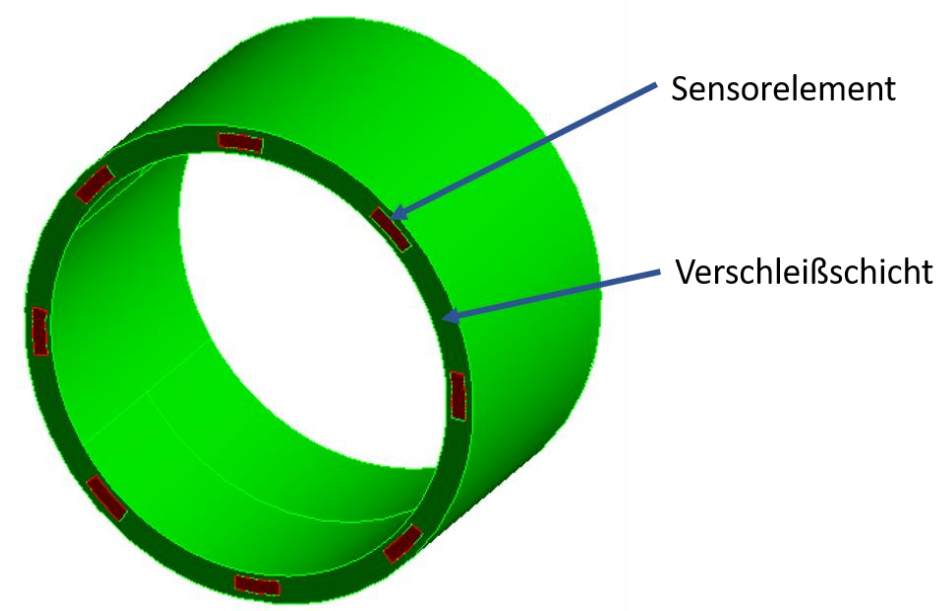

Abbildung 2: Schematischer Aufbau des Kunststoffgleitlagers mit Sensorüberwachung

Die Verschleißschicht, die in direktem Kontakt zur Welle steht, wird mit zunehmender Betriebsdauer verschleißen. Bei ausreichend großem Materialverlust der Verschleißschicht, werden die elektrisch leitfähigen Sensorelemente mit der Welle in Kontakt treten. Die mit einer Spannung beaufschlagten Sensorelemente werden durch die elektrisch leitfähige Welle kurzgeschlossen (Abbildung 3). Damit kann ein Signal erzeugt werden, welches dem Anwender signalisiert, dass das Lager einen bestimmten Verschleißgrad überschritten hat und dementsprechend ausgetauscht werden sollte. Da das elektrisch leitfähige Material ähnliche tribologische Eigenschaften wie die elektrisch nichtleitende Verschleißschicht hat, kann das Lager noch eine gewisse Zeit ohne Probleme weiter in Betrieb bleiben und die Wartung zu einem günstigen Zeitpunkt geplant und durchgeführt werden.

Weiterhin kann im Betrieb bei noch intakter Verschleißschicht die Temperatur mittels einer Widerstandsmessung des elektrisch leitfähigen Materials bestimmt werden. Dazu wird der physikalische Effekt genutzt, dass sich die elektrische Leitfähigkeit mit der Temperatur ändert. Damit können unvorhersehbare Ausfälle, die aus einer Überhitzung des Materials resultieren, vermieden werden. Die temperaturabhängigen Belastungsgrenzen sind materialabhängig und können ermittelt werden. Wird das Kunststoffgleitlager unterhalb dieser Grenze betrieben, ist es zuverlässig sehr lange einsetzbar. Wird die thermische Belastungsgrenze hingegen überschritten, fällt es mit einer bestimmten Wahrscheinlichkeit abhängig von der Last und der Dauer der Temperaturüberschreitung zeitnah aus. 


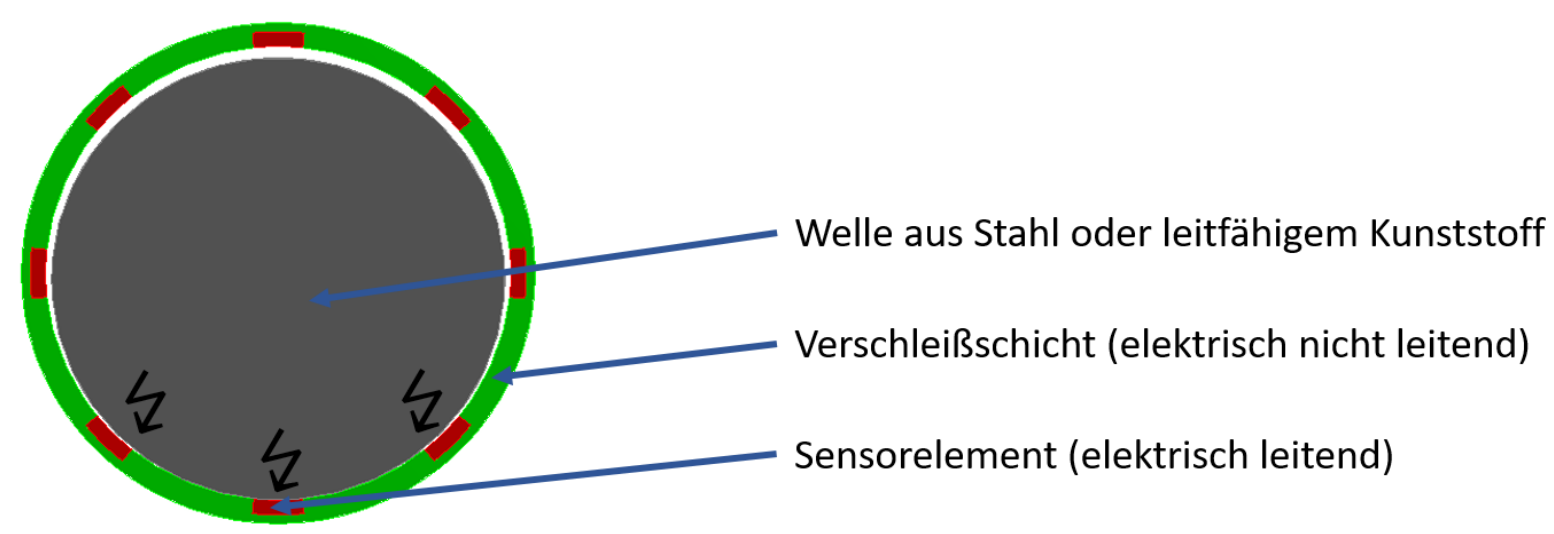

Abbildung 3: Schematische Darstellung des Kunststoffgleitlagers im Verschleißfall (Kurzschluss

\section{Numerische Untersuchungen}

Um ein besseres Verständnis der mechanischen, thermischen und elektrischen Eigenschaften des Systems Gleitlager-Welle zu bekommen, wurden umfangreiche numerische Untersuchungen durchgeführt. Dazu wurde ein Simulationsmodell aus einer Welle, dem Kunststoffgleitlager mit integrierter Verschleißsensorik und einem Lagergehäuse aufgebaut (Abbildung 4). Die Randbedingungen wurden so gewählt, dass das Gehäuse auf der Unterseite fixiert ist. Außerdem wurde die rotatorische und axiale Bewegung der Welle blockiert, um eine stabile Konvergenz der Berechnung zu gewährleisten. In der Mitte der Welle wurde eine Kraft von $60 \mathrm{kN}$ appliziert, welche beispielhaft dem Anwendungsfall entspricht. Um Rechenzeit zu sparen, wurde die Symmetrie des Modellaufbaus ausgenutzt und die Berechnung auf ein Halbmodell beschränkt.

Mithilfe der statisch-mechanischen Berechnung konnten erste Erkenntnisse über das Deformationsverhalten des Systems Gleitlager-Welle gewonnen werden. Dabei wurde ersichtlich, dass es auf der Lagerober- und -unterseite hälftig zur Ausprägung einer Flächenpressung kommt (Abbildung 5 links).

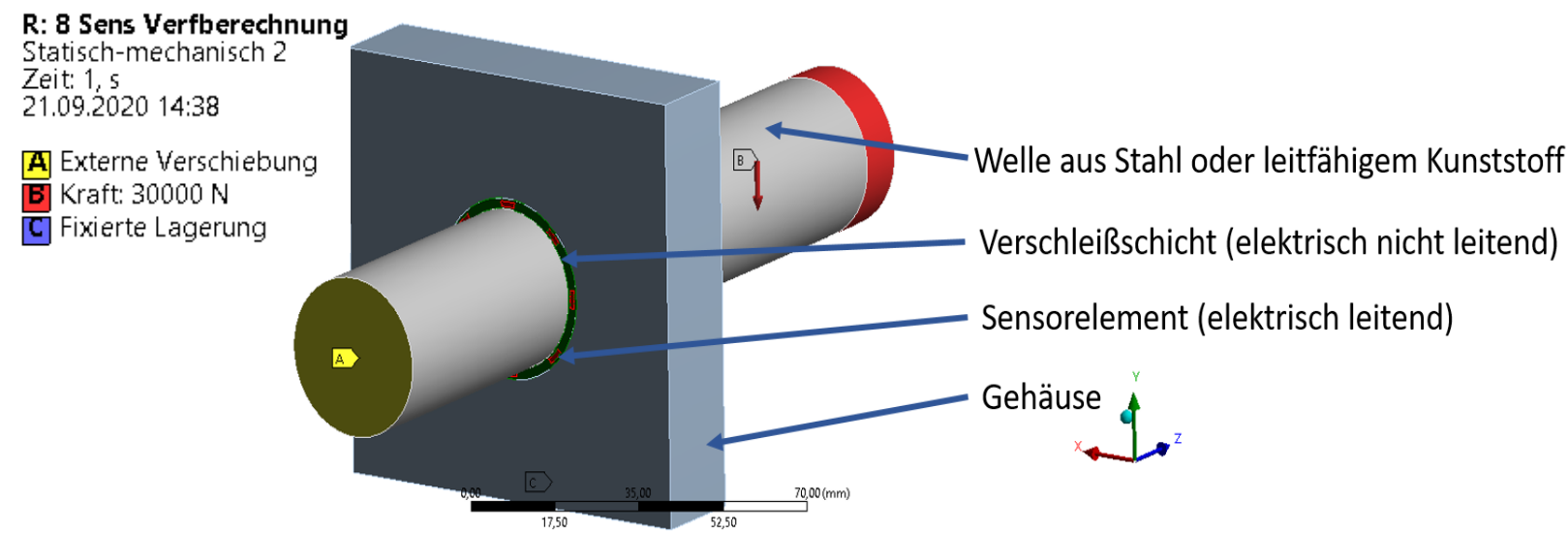

Abbildung 4: Simulationsmodell als Halbmodell mit Randbedingungen 

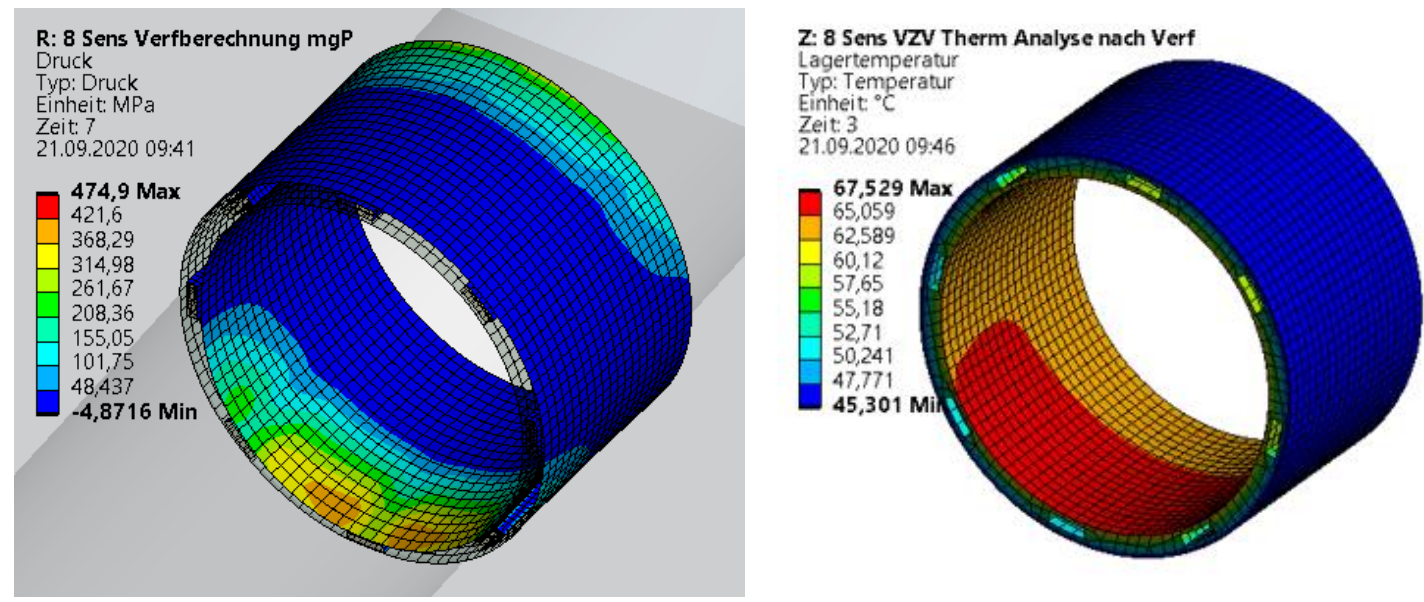

Abbildung 5: Flächenpressung im Lagergrund (links) und Temperaturverteilung im Lager (rechts)

Diese Ergebnisse halfen bei der sich anschließenden thermischen Simulation zur Bestimmung der Gleitlagertemperaturen. Die Flächenpressung definierte in der thermischen Simulation eine Wärmequelle. Außerdem wurden die Oberflächen des gesamten Modells mit einer Konvektionsrandbedingung beaufschlagt, um eine Abfuhr der generierten Wärmeleistung zu gewährleisten. Durch die Variation der Wärmeleistung der Wärmequelle konnten verschiedene thermische Belastungszustände simuliert werden.

Die Simulationsergebnisse zeigen eine Temperaturverteilung mit den beiden Maxima in den Gebieten der Flächenpressungen (Abbildung 5 rechts). Die Lagermaxima und die Sensormittelwerte wurden dazu genutzt, um einen Zusammenhang zwischen messbarem Sensorwert und der realen maximalen Lagertemperatur herzustellen. Dieser Zusammenanhang wurde mittels einer linearen Regression für verschiede Verschleißgrade des Lagers in Abbildung 6 dargestellt.

Weiterhin erfolgte eine numerische Untersuchung des elektrischen Strömungsfeldes. Hierbei sollte der Stromfluss untersucht werden, der im Verschleißfall dem Anwender das Signal zum Austausch des Lagers geben soll. In Abbildung 7 ist dargestellt, dass nach der Erosion der Verschleißschicht, ein elektrischer Strom zwischen mindestens zwei Sensoren

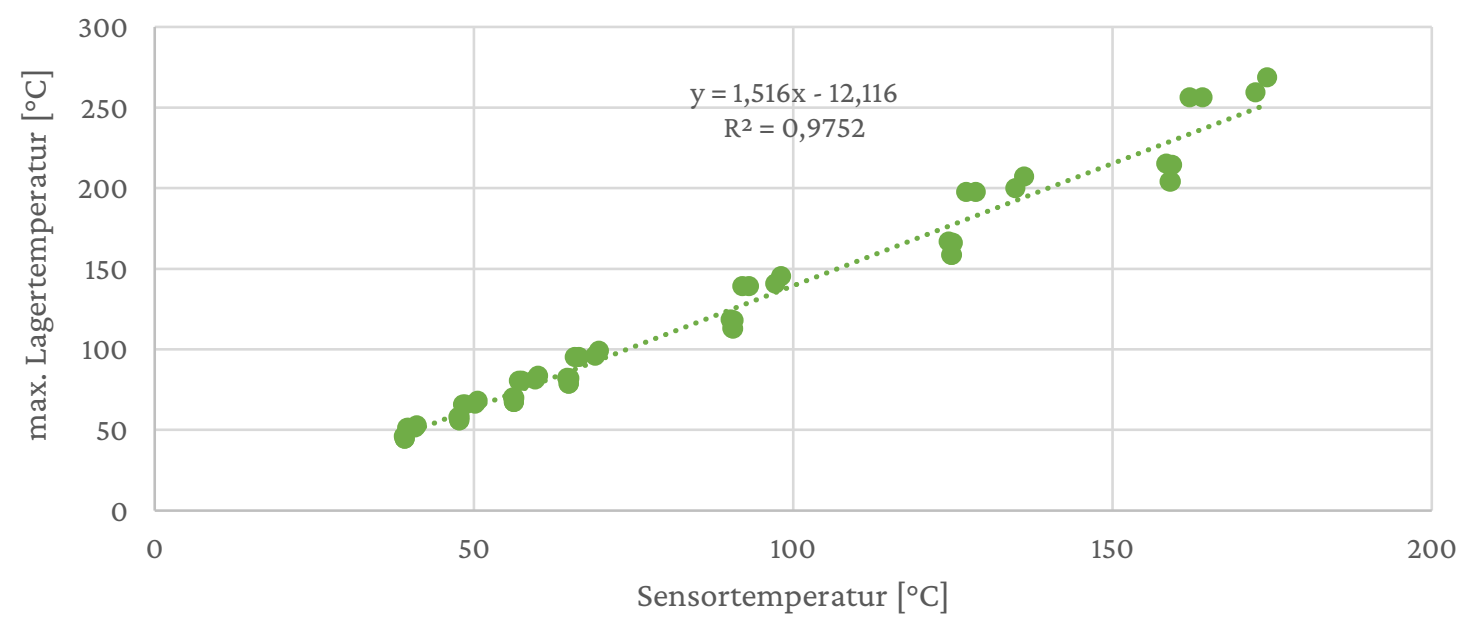

Abbildung 6: Zuordnung der messbaren Sensortemperaturen zu den realen max. Lagertemperaturen 


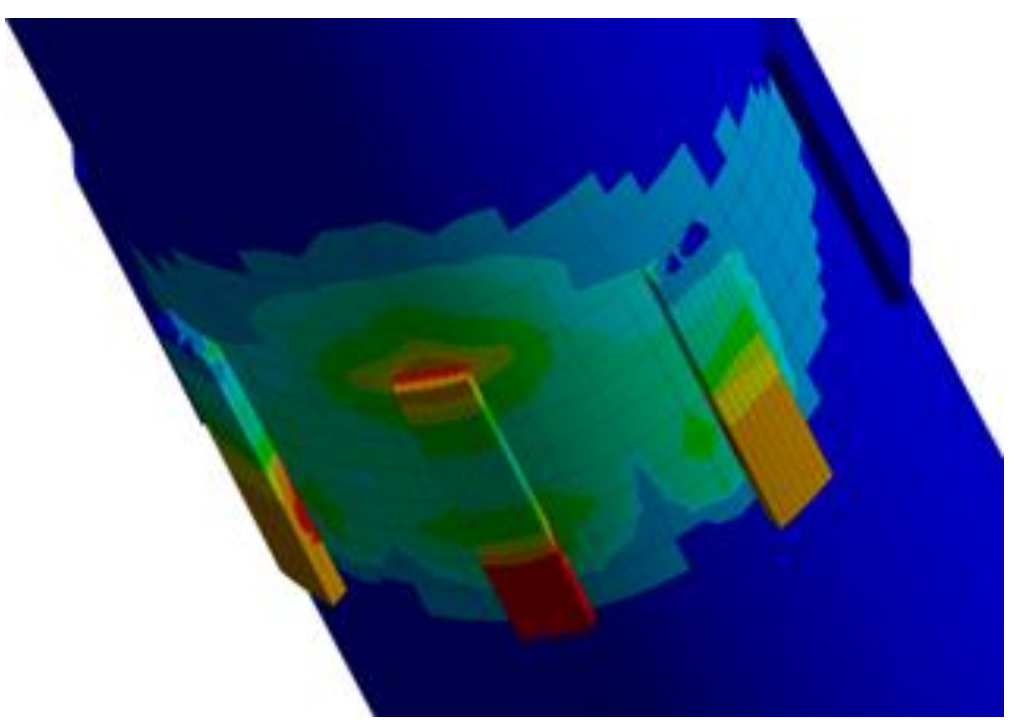

Abbildung 7: Kurzschluss mehrerer Sensoren durch

Wellenkontakt im Verschleißfall

über die Welle fließt. Damit konnte simulativ gezeigt werden, dass das Funktionsprinzip gewährleistet ist.

\section{Experimentelle Untersuchungen}

Um einen Bezug zwischen der Temperatur und dem elektrischen Messsignal der Sensorelemente herstellen zu können, bedurfte es präziser Untersuchungen des Durchgangswiderstands der Sensorelemente. Dabei wurden die Sensorelemente aus vier verschiedenen elektrisch modifizierten Materialien in einer Klimakammer untersucht. Der Messaufbau ist in Abbildung 8 dargestellt.

Die Temperatur wurde dabei mittels eines Thermoelementes gemessen und im Betriebsbereich des Kunststoffgleitlagers variiert. Parallel dazu wurden die Durchgangswiderstände der Sensorelemente bestimmt. Die Messungen zeigten, dass bei einer Temperaturerhöhung auch der elektrische Widerstand der Sensorelemente eine Änderung aufweist. Dieser Zusammenhang ist in Abbildung 9 dargestellt und liefert die Grundlage, um später in

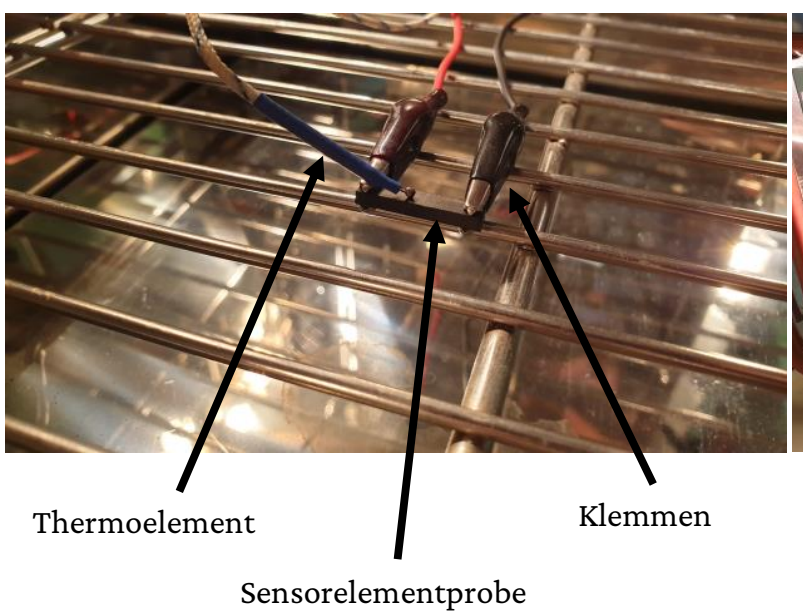

Sensorelementprobe

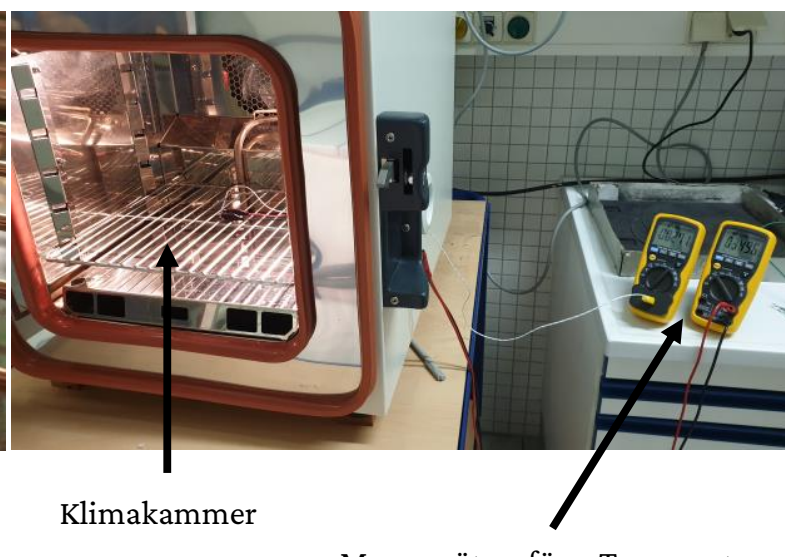

Messgeräte für Temperatur und Widerstand

Abbildung 8: Versuchsaufbau mit Klimakammer 


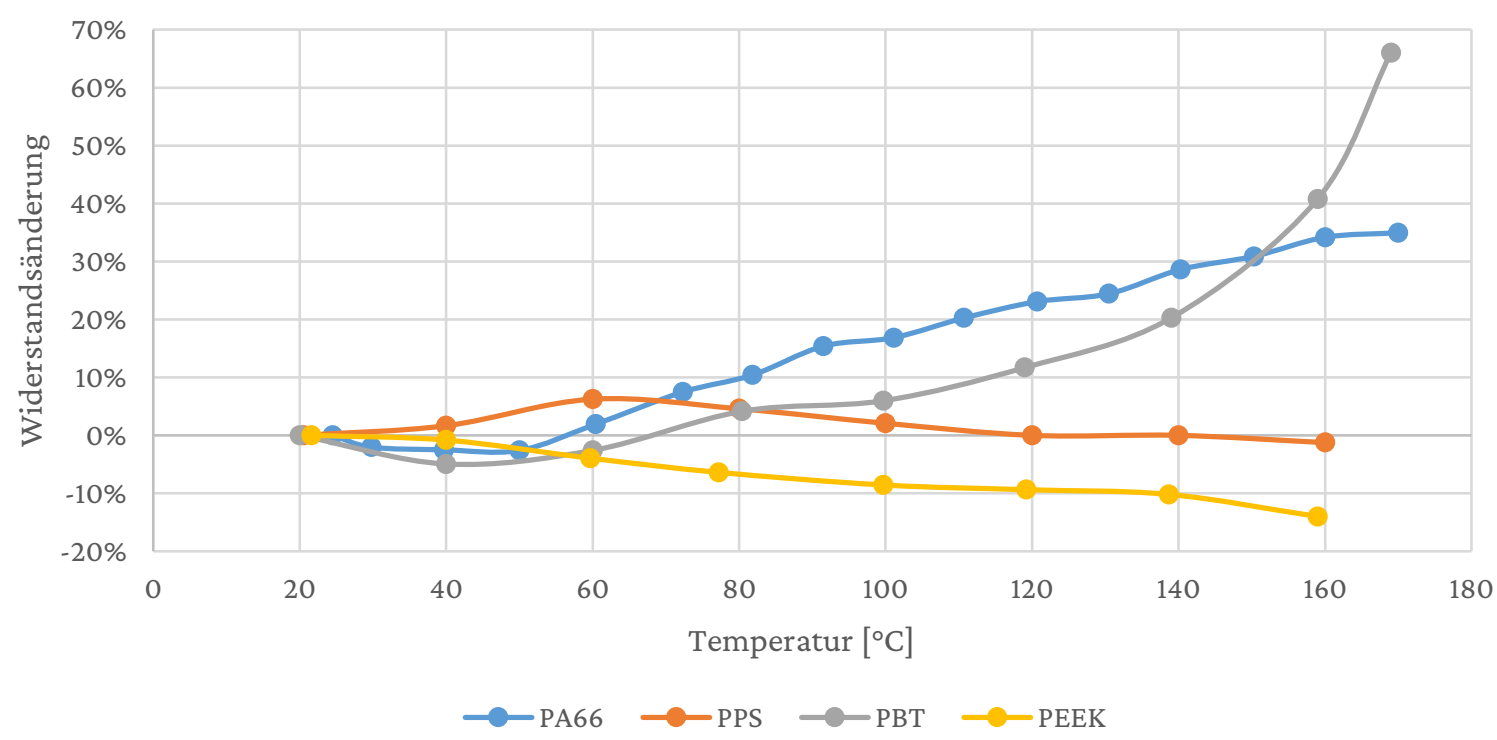

Abbildung 9: Messergebnisse des Durchgangswiderstand der Sensorelemente für verschiedene elektrisch modifizierte Kunststoffe

Echtzeit Aussagen zur Temperatur des Lagers treffen zu können. Besonders positiv ist dabei das Verhalten des Materials PA66 hervorzuheben, da es eine lineare Erhöhung des Widerstands bei einer Temperatur ab $50{ }^{\circ} \mathrm{C}$ aufweist. Auch der Werkstoff PBT würde sich trotz seines exponentiellen Verhaltens eignen. Das Material PEEK zeigt eine nahezu lineare negative Widerstandsänderung bei Temperaturerhöhung. Die Widerstandsänderung ist allerdings sehr gering und damit im Anwendungsfall schwer aufzulösen. Der Werkstoff PPS zeigt hingegen eine hohe Temperaturstabilität hinsichtlich seiner Widerstandsänderung und eignet sich damit nicht für den hier beschriebenen Anwendungsfall.

\section{Auswerteelektronik}

In einem weiteren Arbeitsschritt wurde die Auswerteelektronik an der Professur IMS ausgelegt (Abbildung 10). Das kompakte Modul (Platinenmaße: $35 \mathrm{~mm}$ x $70 \mathrm{~mm}$ ) beinhaltet neben den Messverstärkern auch einen Mikrocontroller, der die Messsignale in die physikalischen Größen umwandelt. Weiterhin ist ein W-Lan Modul integriert, mit dem die Messdaten an eine Empfängereinheit gesendet werden können. Dies kann z. B. ein Computer oder ein Smartphone sein. Später im Entwicklungsprozess soll noch eine App entwickelt werden, um die Daten graphisch ansprechend auf einem Smartphone oder Tablet darzustellen.

Die Funktion (Temperatur- und Verschleißmessung) der Auswerteeinheit wurde durch verschiedene Simulationen nachgewiesen und wird derzeit an der Professur hergestellt. 


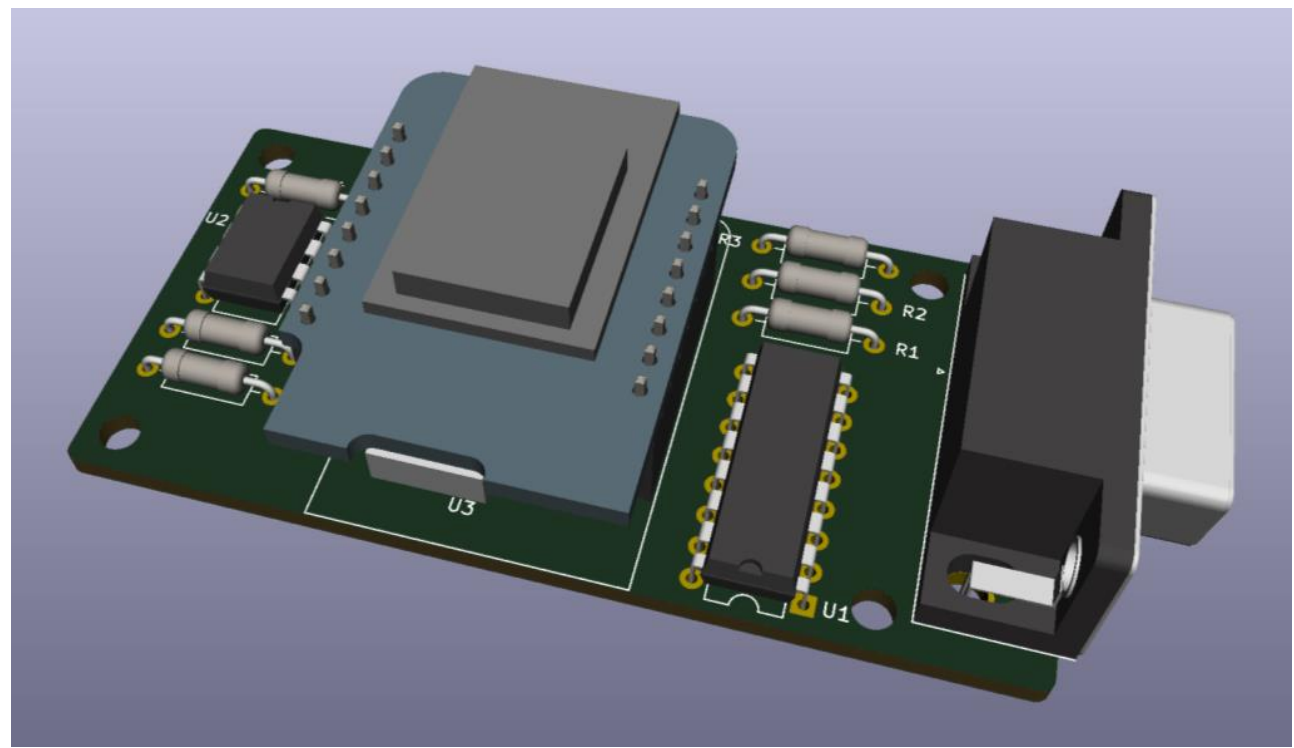

Abbildung 10: Auswerteeinheit

\section{Herstellung eines Funktionsmodells mittels FDM-Druck}

Ein erster Prototyp wurde mittels FDM-Technologie hergestellt. Dabei wurden zunächst Kunststoffe gewählt, die leicht beschaffbar und für den 3D-Druck gut geeignet sind. In der Abbildung 11 ist der funktionsfähige Prototyp dargestellt. Er besteht aus zwei Kunststoffen (einem leitfähigen und einem nicht leitfähigen), die in einem Arbeitsgang aufgetragen werden. Die elektrische Funktion ist bei diesem Lager bereits voll gegeben, die tribologische Funktion aber nur mit Einschränkungen.

Deshalb wird in einem weiteren Schritt ein spezieller tribologisch optimierter Kunststoff für die Gleitflächen und ein leitfähiger Kunststoff, der höhere Temperaturen verträgt, verwendet. Somit sollte dieses Lager aus dem 3D-Drucker nahezu die gleichen Eigenschaften wie ein konventionell hergestelltes Lager aufweisen. Es kann somit auch speziell auf Kundenwünsche ab einer Stückzahl von eins kostengünstig eingegangen werden.

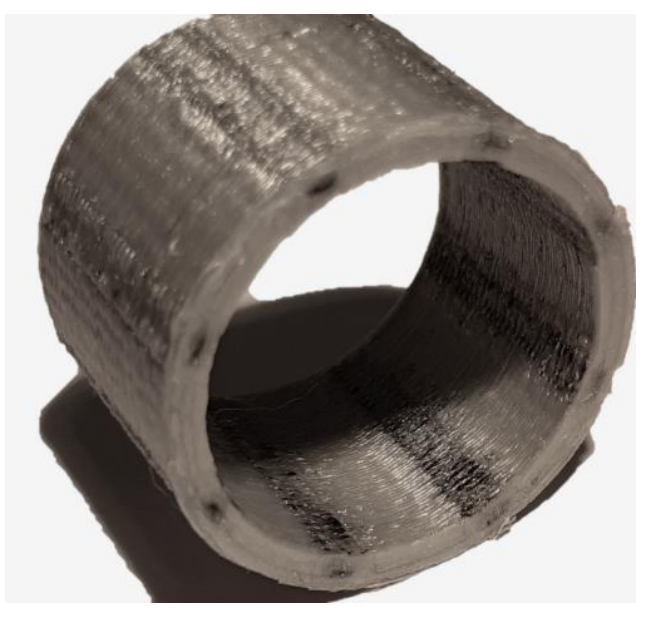

Abbildung 11: Mittels FDM-Druck hergestellter Prototyp des Kunststoffgleitlagers mit integrierter Verschleißsensorik 


\section{Zusammenfassung}

Das im Forschungsprojekt vorgestellte Kunststoffgleitlager mit intelligenter Verschleißsensorik bietet die Grundlage, Wartungsintervalle planbarer $\mathrm{zu}$ machen und Stillstandzeiten oder gar Folgeschäden zu vermeiden. Infolgedessen besitzt es ein enorm hohes Potenzial Kosten zu senken und eine nachhaltigere Instandhaltung zu gewährleisten, welche den vorzeiten Austausch von noch gebrauchsfähigen Lagern vermeidet. Es zeichnet sich daher als ökonomischere und ökologischere Alternative zum herkömmlichen Kunststoffgleitlager aus.

Zusammenfassend konnte mithilfe simulativer und messtechnischer Untersuchungen gezeigt werden, dass das grundlegende Funktionsprinzip zur Messung des Verschleißgrades und der Temperatur gewährleistet ist. Dabei wurde das elektrische leitfähige Material PA66 als besonders geeignetes Sensorelement herausgearbeitet. Ebenso konnte eine Kennlinie zum Verhalten zwischen gemessener Sensortemperatur und realer maximaler Lagertemperatur simulativ bestimmt werden. Weiterhin wurde die Auswerteelektronik entwickelt und ein Prototyp mittels FDM-Druck hergestellt.

Die Ergebnisse können in der weiteren Entwicklung dazu verwendet werden, das Kunststoffgleitlager in einem Spritzgussverfahren serienmäßig herzustellen und damit in Massenproduktion dem Markt zugänglich zu machen. Dafür sind umfangreiche Funktionstest des Sensorgleitlagers notwendig. Weiterhin ist ein Verständnis über das temperatur bedingte Verschleißverhalten der verwendeten Kunststoffe nötig und diese sind hinsichtlich ihrer Zuverlässigkeit und Lebensdauer zu optimieren.

\section{Danksagung}

Das ZIM-Vorhaben ZF 4516707LP9 wurde im Rahmen des Zentralen Innovationsprogramms Mittelstand vom Bundesministerium für Wirtschaft und Energie aufgrund eines Beschlusses des Deutschen Bundestages gefördert. Wir danken den genannten Institutionen für die Bereitstellung der finanziellen Mittel.

\section{Quellen}

[1] https://www.ggbearings.com/de/gleitlager-produkte/solid-polymer (Stand: 18.09.2020)

[2] Valentin L. Popov: Kontaktmechanik und Reibung, Berlin 2009

[3] VTH Verband Technischer Handel e.V.: Predicted Maintenance. In: VTH Ausblicke \#1, page 6 (VTH Verband Technischer Handel e.V., 2018)

[4] https://cdn1.vogel.de/unsafe/540x0/smart/images.vogel.de/vogelonline/bdb/1372900/1372979/original.jpg (Stand: 25.08.2020) 